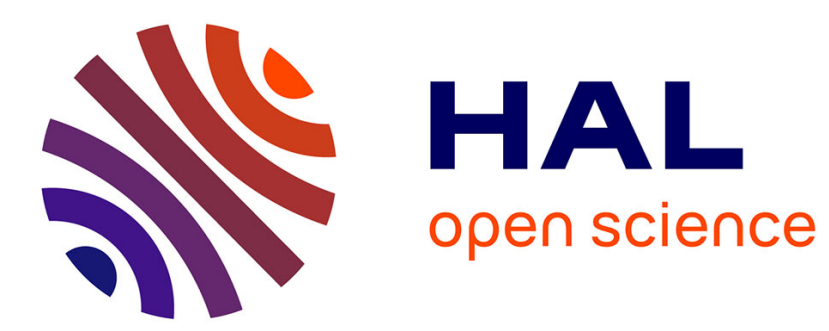

\title{
A PRACTICAL APPROACH TO THE THEORETICAL STRUCTURE OF GRAIN BOUNDARIES
}

\author{
B. Ralph
}

\section{- To cite this version:}

B. Ralph. A PRACTICAL APPROACH TO THE THEORETICAL STRUCTURE OF GRAIN BOUNDARIES. Journal de Physique Colloques, 1975, 36 (C4), pp.C4-71-C4-79. 10.1051/jphyscol:1975408 . jpa-00216313

\section{HAL Id: jpa-00216313 https://hal.science/jpa-00216313}

Submitted on 1 Jan 1975

HAL is a multi-disciplinary open access archive for the deposit and dissemination of scientific research documents, whether they are published or not. The documents may come from teaching and research institutions in France or abroad, or from public or private research centers.
L'archive ouverte pluridisciplinaire HAL, est destinée au dépôt et à la diffusion de documents scientifiques de niveau recherche, publiés ou non, émanant des établissements d'enseignement et de recherche français ou étrangers, des laboratoires publics ou privés. 


\title{
A PRACTICAL APPROACH TO THE THEORETICAL STRUCTURE OF GRAIN BOUNDARIES
}

\author{
B. RALPH \\ Department of Metallurgy and Materials Science, \\ University of Cambridge, England
}

\begin{abstract}
Résumé. - Ce papier résume les modèles théoriques actuels de la structure des joints de grains et essaye de déterminer les possibilités présentées par ces modèles pour définir la structure des joints de grains présents dans les matériaux polycristallins commerciaux. On montre que les modèles permettent une description précise des dislocations intrinsèques du matériau considéré mais, en outre ils permettent de prévoir l'existence d'autres défauts de structure qui ont été mis en évidence ultérieurement.

Abstract. - This paper surveys the current theoretical models of grain boundary structure and attempts to see to what extent they are capable of defining the boundary structure found in commercial polycrystalline materials. It is shown that an adequate description of the intrinsic dislocation content is achieved by such models but that further classes of defect are to be expected and found in commercial materials.
\end{abstract}

1. Introduction. - The real structure of grain boundaries is a topic of great theoretical interest and practical importance. Modern metallic-based materials are normally utilized in technological practice in a polyphase polycrystalline form. In such a form the distribution of phases and of defects is of major importance in controlling material properties. To understand the rôle of grain (or crystal) boundaries in determining properties and the influence they have on the development of microstructure during thermomechanical processing, it is essential to develop a clear picture of grain boundary structure. Further, it is necessary to be able to determine at least some aspects of this structure experimentally.

This article seeks to look, in outline, at the development of theoretical models of grain boundary structure concentrating on findings that have been subject to experimental verification. The aim is to see to what extent current theoretical models are capable of describing the type of grain boundary structure seen in practical polycrystalline materials.

2. Early models. - Current thinking on the structure of grain boundaries perhaps begins with the transition lattice model of Hargreaves and Hill [1]. This model envisages atoms in the boundary region existing in sites which are transitional between sites defining the crystals on either side of the boundary. A considerable number of attempts at refining this model were made, notably amongst them was the island model of Mott [2]. This approach was successfully applied to the under-

Written whilst on leave at Afdeling der Werktuigbouwkunde, Technische Hogeschool Twente, Enschede, Netherlands. standing of a number of boundary dependent processes (e. g. [3]).

Further development of the transition lattice model occurred and in particular efforts were made to apply dislocation models to boundary structure. For the low angle grain boundary there is considerable evidence supporting the Read and Shockley model which envisages these boundaries as comprised of arrays of matrix dislocations. However, for high angle grain boundaries the difficult problem of core overlap precluded extensive development of such models [4].

In essence, the early approaches to grain boundary structure considered all high angle boundaries as having similar structure regardless of the relative orientation of the two grains and the plane of the boundary. Consideration of the crystallographic parameters of the boundary has lead to major developments in the theoretical treatment of boundaries. These developments are outlined in the next sections.

3. Coincidence. - A first stage in the understanding of boundary geometry came when it was realised that certain angular misorientations about specific axes of misorientations lead to the formation of a lattice of coincident points common to the two grains [5]. This resultant coincident site lattice extended across any grain boundary. Relatively early on, it was shown that the presence of boundaries in coincidence orientations could lead to properties which differed from those of other boundaries (e.g. [6]). It became common to refer to boundaries as special or general depending on the degree of coincidence between the abutting grains.

A further aspect of coincidence was recognized 
when the orientation of the boundary plane with respect to the coincidence site lattice was considered. Brandon et al. [7] suggested that the boundary when following planes of high density in the coincidence site lattice might show good fit and demonstrated this aspect by some preliminary field-ion experiments. From this idea of boundary plane coincidence they also predicted the presence of grain boundary ledges and suggested that small angular deviations from perfect coincidence might be allowed for by introducing sub-boundaries of dislocations $[7,8]$. The idea of boundary coincidence was greatly extended by Bishop and Chalmers in their coincidence ledge approach [9], and experimental observations of dislocations in the coincidence site lattice were made by Ishida [10].

These approaches considered the macroscopic crystallography of the boundary but did not involve detailed considerations of the boundary structure after relaxation. Further, an approach based on the coincidence of lattice sites is discontinuous with misorientation and what is sought is a model capable of describing the structure of all grain boundaries. In essence the aim is to generalise the type of treatment initially produced for special boundaries.

4. The Bollmann approach. - The most mathematically elegant and detailed treatment of grain boundary structure arose from considerations by Bollmann [11, 12]. Bollmann extended the idea of coincidence of lattice points to coincidence of any points within the cells of the two grains and thus produced his « $O »$ lattice of coincident interstitial points. Here the coincidence site lattice is a natural special case of this approach. In order to treat the most general cases by this technique it proved essential to work with reduced components of the « $O \gg$ lattice where « $O »$ points with similar pattern surrounds were considered.

This formulation essentially sees $« o »$ points as centres of regions of match or fit with regions (or cell walls) between them being misfit regions. In some cases it is anticipated that this misfit would be taken up by primary dislocations whose lines would be the intersection of the cell walls with the boundary plane. Further dislocations (referred to as secondary intrinsic type) would be expected in the general case and these would have small Burgers vectors which are partials of the nearest coincidence site lattice. These secondary intrinsic dislocations maximise the registary between the two crystals at the boundary and are also referred to as D. S. C. (Displacement Shift Complete) dislocations.

The Bollman approach is much the most general and, in particular, it stresses the periodic nature of the grain boundary structure. Much evidence from transmission electron microscopy of precisely oriented bicrystals supports this overall model (e. g. [13, 14]). However, to apply this model the boundary orientation parameters must be known with great precision and such precision is often not possible in studies of polycrystalline material.

5. The structural unit model. - The structural unit model envisages the boundary structure as made up of repeating units (e. $g$ [15]) ; the size of the repeating pattern becoming smaller as a coincidence orientation is approached. In essence the starting point for the structural unit approach is the type of coincidence ledge structure envisaged by Bishop and Chalmers [9]. The structural unit model then considers the relaxation of this structure and in particular allows the computation of this relaxation (e. g. [16-20]). An alternative dynamic computation of grain boundary structure has recently been made [21].

6. Planar matching model. - Pumphrey was the first to suggest that boundary structure might be related to the match/mismatch of plane stacks across the boundary [22]. Subsequently this model was developed and evidence produced for its support [23].

The starting point for this model is the acceptance that the axis of misorientation is a common direction in the two crystals. In all but the triclinic system there is a multiplicity of choice of axis of misorientation but normally an axis can be chosen which is normal or nearly so to a low index stack of planes. If the axis of misorientation is precisely normal to such a set of planes then the stacks on either side of the boundary will be accurately parallel (Fig. 1a). If the axis deviates from such a configuration it is equivalent to saying that these plane stacks are no longer precisely parallel and the resulting small deviations can be considered as the superimposition of small twist components (Fig. $1 b$ ) and/or, small asymetric tilt components (Fig. 1c). These small twist and tilt components bring the plane stacks on either side of the boundary into disregistrary and where this disregistrary is small it can be accommodated by the formation of dislocations with in planer Burgers vectors (Fig. 1d) ; analogous to those proposed by Frank and Van der Merwe for two-phase interfaces (see [23]).

The planar matching model is a particularly appropriate way of analysing data from highly textured polycrystalline aggregates. Further, it is possible to consider the intersection of more than one set of plane stacks at the boundary and as a result derive a complete description of the geometrical mismatch [23]. From this it is then possible to predict the manner in which this mismatch will relax and, as in the case of the Bollmann approach, the general result will be three sets of intrinsic dislocations.

It is perhaps important to realise that the planar matching model is equivalent to the Bollmann 

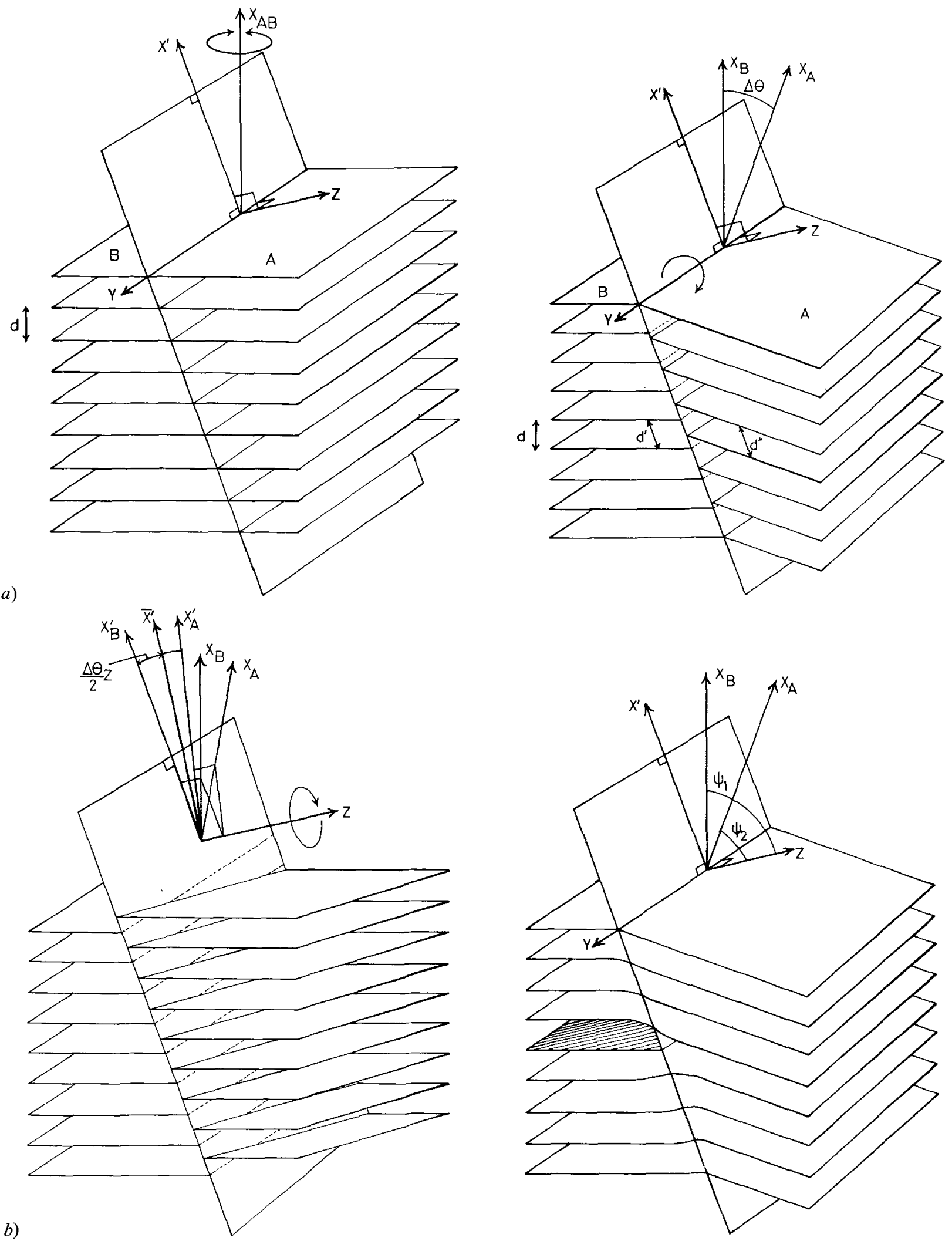

d)

FIG. 1. - Schematic representation of part of the planar matching model. The primary plane stacks on both sides of the boundary are assumed to be smooth structureless planes: a) gives the situation when the normal to both plane stacks is precisely parallel to the axis of misorientation ; $b$ ) shows the unrelaxed effect of introducing a small twist mismatch to the stacks about an axis $(Z)$ normal to the boundary plane. Here the relaxation process could lead to the formation of arrays of screw

dislocations; c) shows the unrelaxed effect of introducing a small asymmetric tilt to the stacks. The result is that at the boundary only certain planes are brought into registrary; d) shows a possible relaxation pattern for the situation shown in figure $1 c$ where the mismatch has been localised to form an intrinsic dislocation of the Van der Merwe type (from reference [23]). 
description (Howell, Jones, Horsewell and Ralph unpublished). The advantages it has over the Bollmann approach in the practical case is that it can be more directly related to the imaging modes of the transmission electron and field-ion microscopes (e. g. [24]). Further, at least a first stage in the analysis by this technique requires much less crystallographic precision in the analysis.

7. Grain boundary structure in practical materials. - The discussion thus far has considered, in the main, the geometrical components of grain boundaries in an equilibrium configuration. The various descriptions proposed all suggest that certain components of the geometrical mismatch can be reduced or relaxed to give a pattern of intrinsic dislocations with small Burgers vectors. Here the descriptions by Bollmann or by the planar matching model lead to the same description. It can also be shown that the coincidence ledge and structural unit descriptions yield similar descriptions. For a planar section of boundary these intrinsic dislocations will be expected to be periodic; in this way the overall strainfields will be reduced.

In practical materials other non-equilibrium components will have to be considered. These components will arise due to the interaction of other grain boundaries (i. e. arise from the fact that the polycrystal is a three-dimensional array of grains); due to the interaction of other defects (principally matrix dislocations) with the grain boundaries; and due to the interaction of solutes and impurities with the grain boundaries.

Thus, in addition to arrays of intrinsic dislocations, a grain boundary in a polycrystalline aggregate will be expected to contain other defect structures. Topographical discontinuities such as grain boundary nodes, changes in grain boundary plane, steps and ledges will be expected. The steps and ledges may arise due to massive slip interaction with the boundary, may represent an attempt on behalf of the boundary to follow low energy planes or may represent a frozen-in stage of grain boundary migration.

Interaction of matrix dislocations with a grain boundary is a complex process. Recently a number of studies of this process have been made (e. g. [25, 26], Howell, Jones, Horsewell and Ralph (unpublished)). This interaction, of a matrix dislocation with a grain boundary, leads to the formation of one or more extrinsic dislocations. These extrinsic dislocations have the same Burgers"vectors as the underlying intrinsic dislocations but longer range strain fields because they perturb the periodicity of the intrinsic structure (this is manifested by increased contrast in an electron microscope image). Extrinsic dislocations are formed when dislocations run-in or are driven into the grain boundary during deformation and have an important rôle in the mechanism of recrystallisation (Howell, Jones and Ralph (unpublished)). Extrinsic dislocations are important in the mechanism of grain boundary sliding [25] and have been shown to be preferential sites for the precipitation of carbides in austenitic steels [27].

To these geometrical defects must be added those arising from the interaction with solutes. Thus a grain boundary in a practical polycrystalline material is expected to be comprised of the following defect types :

a) intrinsic dislocations

b) extrinsic dislocations

c) topographical discontinuities

d) precipitates and other solute interactions.

We might reasonably expect the presence of solute to stabilize the non-equilibrium components of the boundary structure.

It remains to determine what percentage of boundaries in a polycrystalline aggregate would be expected to show this type of structure. On the basis of random orientations, theoretical predictions have been made as to the percentage of boundaries which might be expected to show structure (e. g. [8, 28]). These theoretical predictions suggest that a relatively low percentage of structured boundaries should be encountered. However, a recent series of experimental observations showed that in a particular material and state $78 \%$ of the boundaries showed structure which could be resolved in the electron microscope [29]. It seems that the percentage of boundaries exhibiting structure is sensitively related to the degree of texture in the material and preliminary experiments to determine this inter-relation are in progress [30].

In the remaining section the experimental observations of boundary structure using transmission electron microscopy are considered.

8. Experimental observations of grain boundary structure. - In order to reveal both the equilibrium and non-equilibrium components of grain boundary structure using transmission electron microscopy, a number of imaging modes must be used [31]. Of the available imaging modes weakbeam, boundary diffraction and double diffraction are particularly useful in establishing periodic and non-periodic components. The results of applying such techniques to the study of grain boundary structure in a commercial austenitic steel have been reported [29]. In the remainder of this section, consideration is given to the structures observed in a variety of materials in a number of thermomechanical states.

Figure 2 is a bright-field electron micrograph from a specimen of niobium stabilized $20 \%$ chromium $25 \%$ nickel austenitic stainless steel. A faceted boundary is apparent and on this boundary 


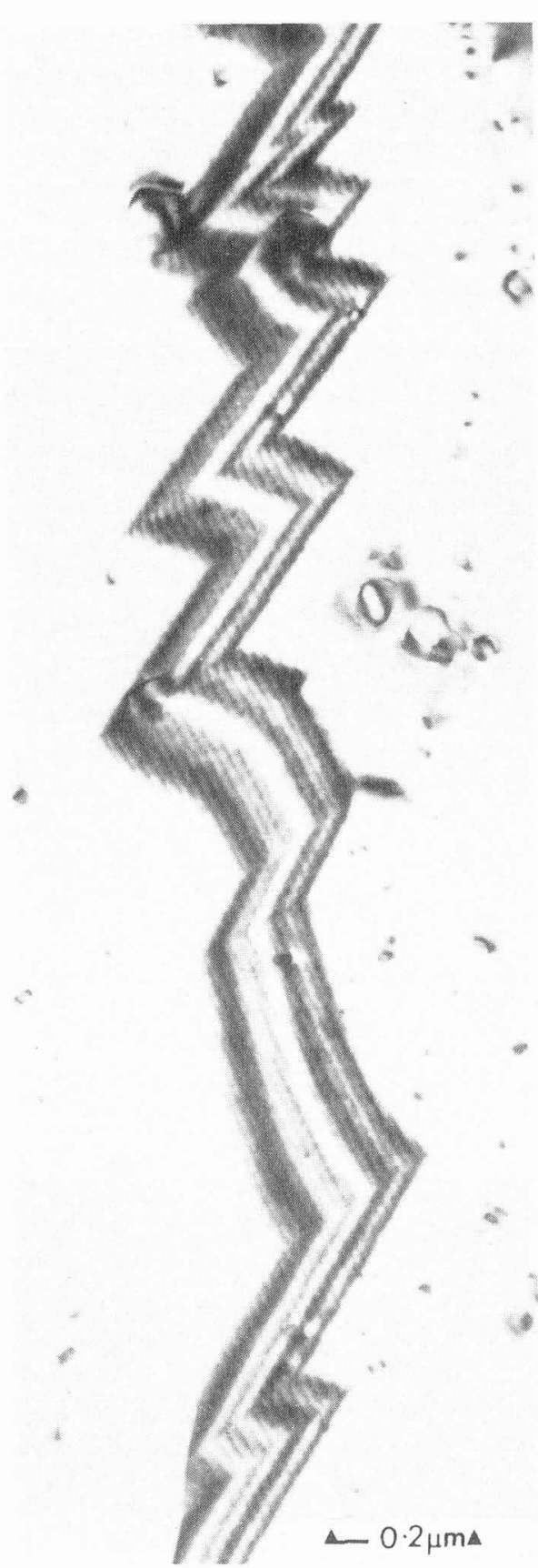

FIG. 2. - Transmission electron micrograph of a faceted boundary in a niobium stabilized $20 \%$ chromium $25 \%$ nickel austenitic steel. An array of Moiré fringes is apparent due to the beating between the nearly parallel $(311)_{A}$ and $(222)_{B}$ planes (Courtesy Jones A. R.).

an array of Moiré fringes is seen. These fringes are formed due to the "beating " between the nearly parallel $(311)_{A}$ and $(222)_{B}$ planes. The fringes do not directly reveal a component of the relaxed structure but are useful in determining overall components of the geometry.

Figure 3 is from a partially recrystallised sample of tungsten lamp filament wire. This micrograph, one of a sequence taken from this particular boundary in a variety of operating diffraction conditions, is part of an overall study aimed at

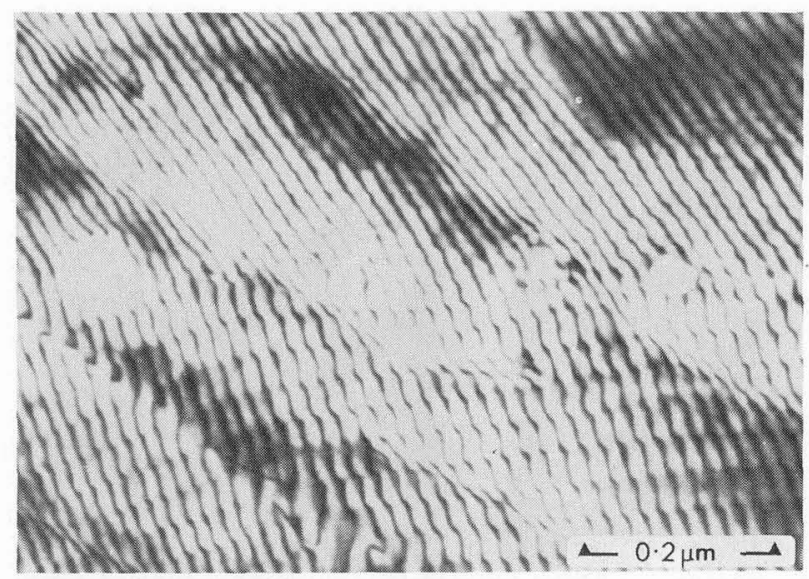

FlG. 3. - Micrograph from a partially recrystallised tungsten specimen. An array of intrinsic dislocations and a row of small voids pinning the boundary is apparent (Courtesy Fleet D. E. and Howell P. R.).

determining the origins of voids which lead to the failure of tungsten lamp filaments [32]. In this micrograph one of the sets of grain boundary dislocations is clearly apparent as is a row of small voids pinning the boundary.

Figure 4 is from a study of the recrystallisation process in.stabilized austenitic stainless steels [33].

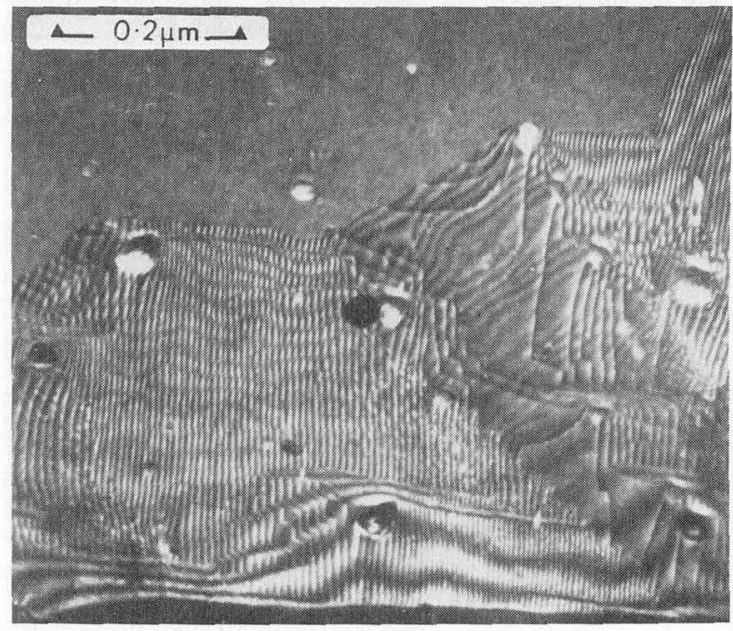

FIG 4. - Weak-beam micrograph of an interface between recrystallised (upper) and unrecrystallised (lower) grains in 20/25 stainless steel. The complex topography, largely due to pinning by a fine dispersion of niobium carbide, is revealed by the change in intrinsic dislocation spacing from one plane segment to another (Courtesy Howell P. R.).

This micrograph was taken in the weak-beam imaging mode for the upper recrystallised grain leaving the lower unrecrystallised grain out of contrast. The intrinsic dislocations are clearly resolved and the complex topography, largely due to interaction with small precipitates, is clearly seen from the way the intrinsic dislocation spacing varies from one plane segment to another. Another aspect of this study of the mechanism of recrystallisation is revealed in figure $5 a$ and $5 \dot{b}$, which show, in 
a)

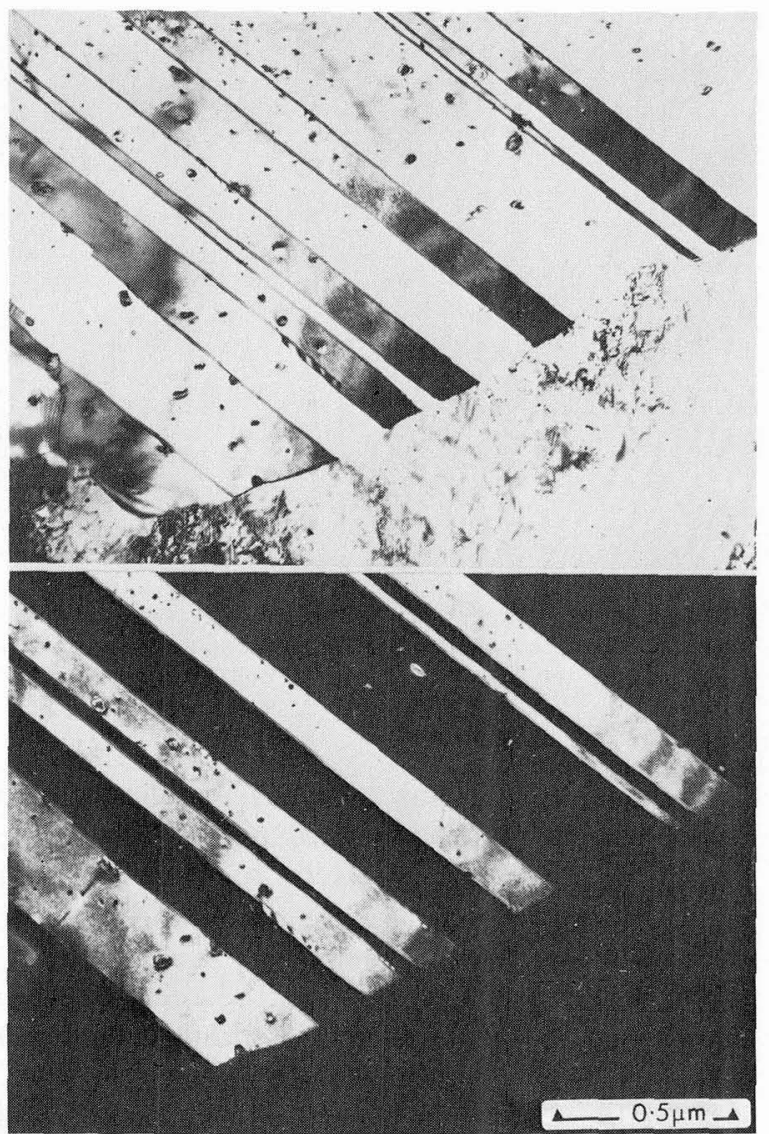

FIG. $5 a$ and $b$. - Bright-field ( $a$ ) dark-field $(b)$ pair of single variant back twinning from a recrystallising interface in 20/25 stainless steel (Courtesy Howell P. R.).

bright-field and centered dark-field respectively, single variant back twinning into the recrystallised material from a boundary between recrystallised and unrecrystallised material. Complex topography at the head of an advancing annealing twin, formed during recrystallisation, is shown in figure 6.

Figure 7 gives a clear indication of the formation

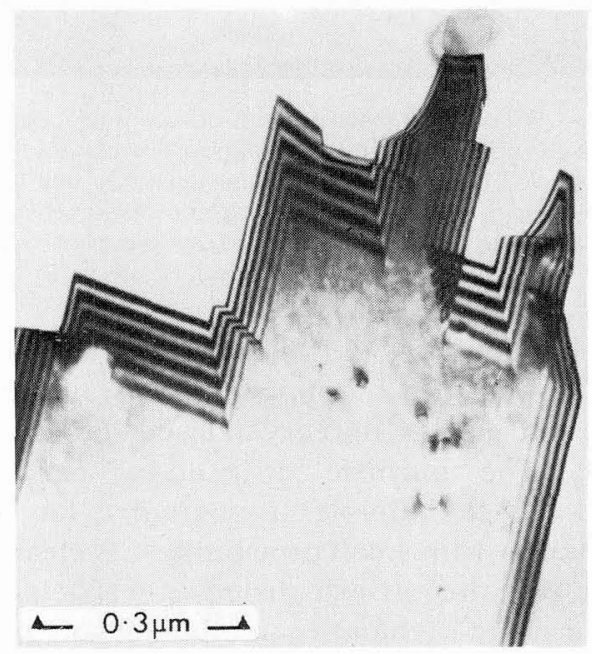

FIG. 6. - Faceted annealing twin formed during recrystallisation of 20/25 stainless steel (Courtesy Howell P. R.). of extrinsic dislocations in austenitic stainless steels. A large carbide on the left-hand side of this micrograph is acting as a dislocation source and the resultant dislocations, seen in bright contrast in this weak-beam micrograph, run towards and interact with the boundary. Each matrix dislocation leads to the formation of two extrinsic dislocations which were then seen to glide along the boundary.

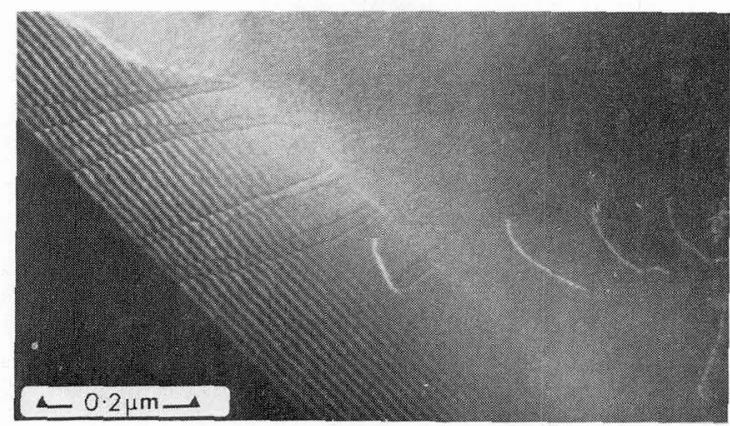

FIG. 7. - Weak-beam image illustrating the formation of extrinsic dislocation by dissociation of matrix dislocations in a $20 / 25$ stainless steel specimen. Each matrix dislocation from the precipitate on the left-hand edge of the matrix produces two extrinsic dislocations which then glide along the boundary (Courtesy Howell P. R. and Kilvington I. T.).

Figure $8 a$ shows an array of extrinsic dislocations in a dark-field micrograph from a stabilized austenitic steel specimen. Figure $8 b$ shows a centered dark-field micrograph using a niobium carbide relection of a boundary in a similar specimen. It is

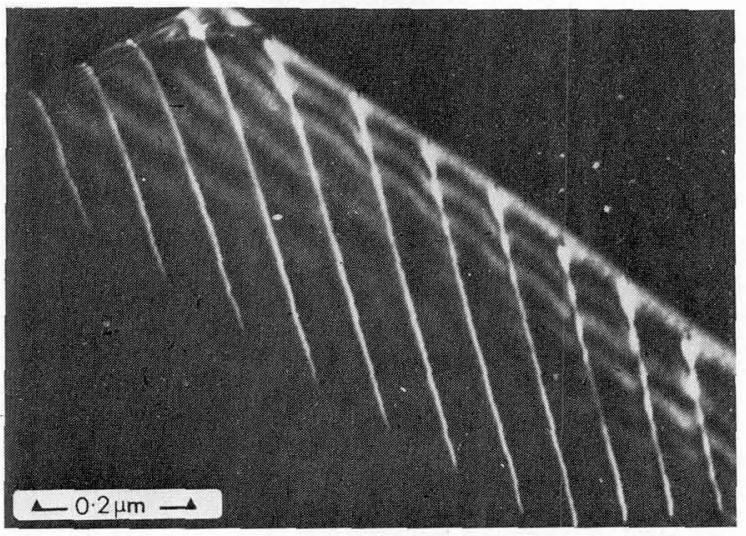

a)

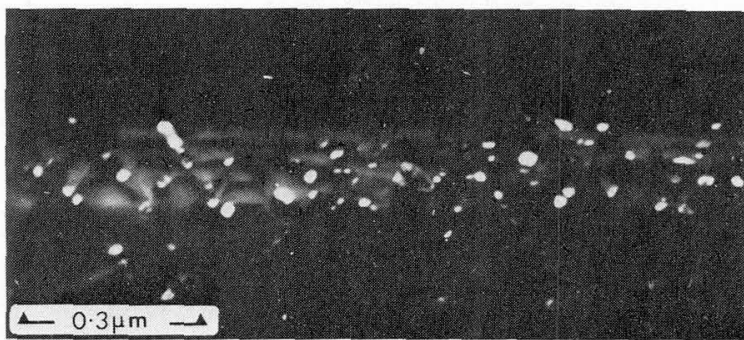

b)

FIG. 8a. - Matrix dark-field micrograph of an array of extrinsic dislocations in a 20/25 stainless steel specimen. $8 b$. - Precipitate dark-field micrograph of the distribution of niobium carbide precipitates at a grain boundary in a 20/25 stainless steel specimen. It can be seen that these precipitates are decorating extrinsic dislocations (Courtesy Howell P. R.). 
relatively easy to show that extrinsic dislocations (as here) and topographical discontinuities are the major sites for precipitation [27].

The micrographs presented above demonstrate that the structure of grain boundaries can be thought of in terms of the simple classification of defect types given in section 7. A current interest is the rôle of grain boundary structure in phase transformations and twinning.

Initially this study was aimed at a theoretical evaluation of the rôle grain boundary dislocations might play in the martensitic transformation from f. c. c. $\rightarrow$ h. c. p. in cobalt-base alloys. Here it was

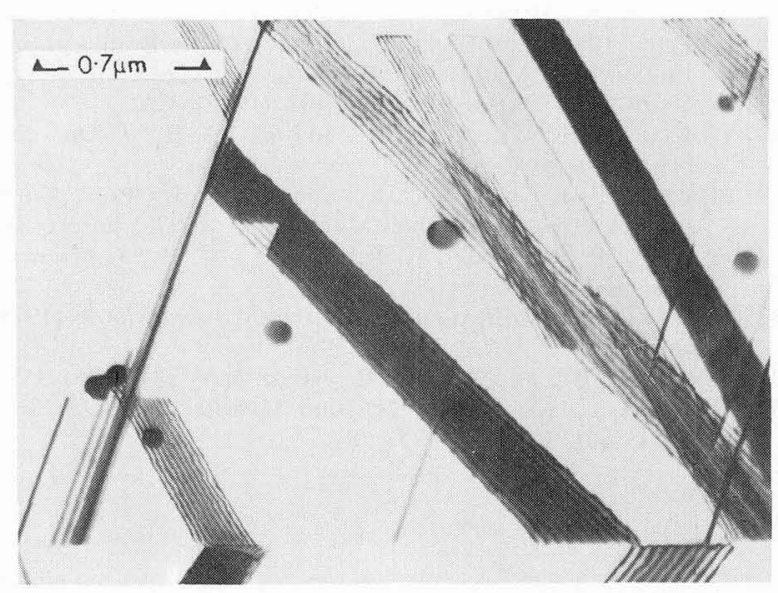

a)

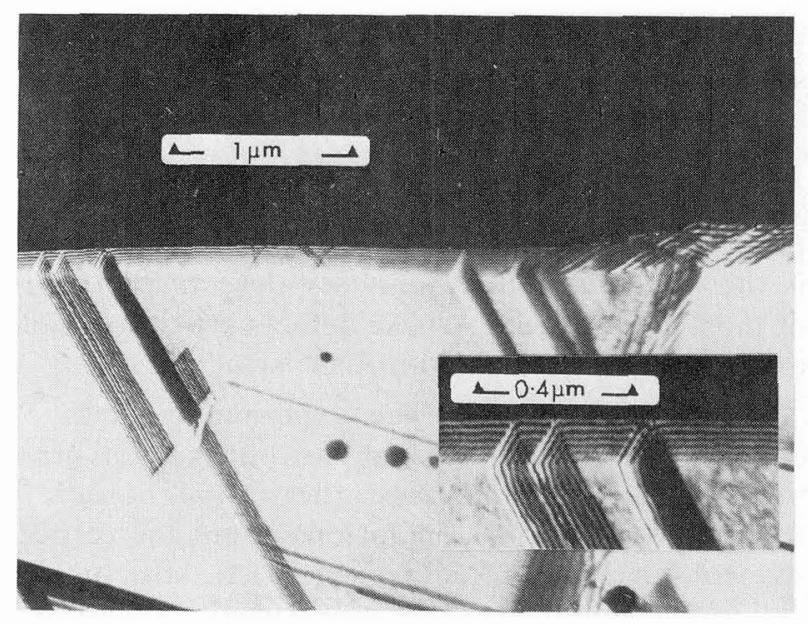

b)

FIG. 9. - Micrographs from partially transformed samples of a cobalt $0.25 \mathrm{wt} \%$ boron alloy; a) showing the nucleation of stacking faults at twin boundaries in the f.c.c. phase : b) showing nucleation of stacking faults at an f. c. c. grain boundary. The insert shows, at higher magnification, that the retained partials at the beginning of the fault are parallel to the intrinsic boundary dislocations (Courtesy Horsewell A.). shown that the necessary partial dislocation arrays could be produced by suitable combinations of grain boundary dislocations [34]. A further experimental stage of this investigation is now under way and figures $9 a$ and $9 b$ are from this study. Both these micrographs are from partially transformed cobalt 0.25 wt $\%$ boron samples. Figure $9 a$ shows the nucleation of stacking faults at twin boundaries in $\mathrm{f}$. c. c. material. Figure $9 b$ shows the nucleation of stacking faults at a grain boundary in f.c. $c$. material and on the insert it can be seen that the retained partials at the start of the faults are parallel to the intrinsic boundary structure.

9. Discussion and conclusions. - The theoretical models of grain boundary structure now available give a relatively clear picture of the type of structure to be expected in boundaries in an « equilibrium» configuration. These models all predict that some percentage of boundaries will show a periodic arrangement of intrinsic dislocations. As yet these theoretical models are insufficiently refined to determine the full pattern of relaxations expected and it is also true to say that no experimental technique has the required resolution to resolve all these relaxations. It is to be hoped that refinements in the area of computer modelling will add important information in this area.

Whilst our picture of the idealized boundary, say between crystals in an especially grown bicrystal, is relatively complete, the structure of boundaries in real polycrystalline materials is only just beginning to receive attention. This paper has attempted to show that boundaries in this case will contain not only the equilibrium components (intrinsic structure) but will also contain non-equilibrium components as well. These non-equilibrium components may just be a transitory feature (although in their own right very important in determining for instance grain boundary sliding) or they may be stabilized by solutes and precipitates.

Acknowledgements. - The author is grateful to Professor R. W. K. Honeycombe for the provision of laboratory facilities. Financial support from the Science Research Council, the United Kingdom Atomic Energy Authority (Harwell and Springfields laboratories), and Thorn Lighting Ltd., is gratefully acknowledged. The author also wishes to thank Dr. P. R. Howell, Dr. A. R. Jones, Mr. D. E. Fleet and $\mathrm{Mr}$. A. Horsewell for valuable discussions. 


\section{References}

[1] Hargreaves, F., and Hill, R. J., J. Inst. Met. 41 (1929) 257

[2] MotT, N. F., Proc. Phys. Soc. A63 (1950) 616

[3] MCLEAN, D., Grain Boundaries in Metals (Clarendon Press : Oxford) 1957.

[4] LI, J. C. M., J. Appl. Phys. 32 (1961) 525.

[5] Kronberg, M. L., and Wilson, F. H., Trans. A.I.M.E. 185 (1949) 501.

[6] AUST, K. T., in Recovery and Recrystallisation of Metals Ed. L. Himmel (Interscience Publishers: N. Y.) 1962, p. 131 .

[7] BRandon, D. G., RALPH, B., Ranganathan, S., and WALD, M. S. Acta Met. 12 (1964) 813.

[8] Brandon, D. G., Acta Met. 14 (1966) 1479

[9] Bishop, G., and CHAlmers, B., Scr. Metall. 2 (1968) 133.

[10] ISHIDA, Y., J. Mat. Sci. 7 (1972) 72.

[11] Bollmann, W., Crystal Defects and Crystalline Interfaces (Springer-Verlag : Berlin 1970).

[12] Bollmann, W., J. Microsc. 102 (1974) 233.

[13] Schober, R., and Balluffi, R. W., Phil. Mag. 21 (1970) 846.

[14] KegG, C. R., Horton, C. A. P., and Silcock, J. M., Phil. Mag. 27 (1973) 1041.

[15] Gleiter, H., Phys. Stat. Sol. (b) 45 (1971) 9.

[16] Weins, M. J., Chalmers, B., Gleiter, H., and Ashby, M., Scr. Metall. 3 (1969) 601 .

[17] Baroux, B., Biscondi, M., and Goux, C., Phys. Stat. Sol. (a) 2 (1970) 415 .

[18] Hasson, G. C., Gulllot, J. B., Baroux, B., and Goux, C., Phys. Stat. Sol. (a) 2 (1970) 551.
[19] Johannesson, T., and Tholen, A., Phil. Mag. 21 (1970) 1223.

[20] Weins, M. J. Gleiter, H., and Chalmers, B., J. Appl. Phys. 42 (1971) 2639.

[21] Cotterill, R. M. J., LefFers, T., and Lillolt, H., Phil. Mag. 30 (1974) 265.

[22] Pumphrey, P. H., Scr. Metall. 6 (1972) 107.

[23] Ralph, B., Howell, P. R., and Page, T. F., Phys. Stat. Sol. (b) 55 (1973) 641.

[24] Howell, P. R., Page, T. F., and Ralph, B., Phil. Mag. 25 (1972) 879 .

[25] Horton, C. A. P., and Silcock, J. M., J. Microsc. 102 (1974) 339.

[26] Pumphrey, P. H., and Gleiter, H., J. Microsc. 102 (1974) 349.

[27] Jones, A. R., Howell, P. R., Page, T. F., and Ralph, B., Proc. 4th Bolton Landing Conf., on Grain Boundaries in Engineering Materials, (1974) (in press).

[28] Warkington, D., J. Microsc. 102 (1974) 301.

[29] Howell, P. R., Jones, A. R., and Ral.PH, B., J. Mat. Sci. (1975) (in press)

[30] Howell, P. R., Fleet, D. E., and Ralph, B., Proc. Conf on Textures (Cambridge) Metals Soc., (1975) (in press).

[31] Howell, P. R., Jones, A. R., and Ralph, B., J. Microsc. 102 (1974) 323.

[32] Fleet, D. E., and Ralph, B., J. Less Comm. Metals (1975) (in press).

[33] Jones, A. R., and RalPh, B., Acta Met. 23 (1975) 355.

[34] Horsewell, A., RAlPH, B., and Howell, P. R., Phys. Stat. Sol. (a) 29 (1975) 587

\section{DISCUSSION}

D. A. SmIrH : Dr. Ralph did not give any crystallographic data to characterise the boundaries he described. Can we conclude that for the experiments described the important factor is that dislocations exist in grain boundaries and that the details of the structure do not exert a profound influence.

B. RALPH : Basically the answer to your question is yes. Obviously we have some crystallographic data for all the boundaries we have studied. However, only in very favourable circumstances are we able to measure the main five crystallographic boundary parameters with sufficient precision to match these to the density and burger's vectors of the intrinsic (D.S.C.) dislocations.

My main thesis is two-fold :

a) The vast majority of boundaries are seen to contain intrinsic structure. When we are able to make sufficiently precise crystallographic measurements then the arrays of dislocations seen are as would be predicted from an $« 0 » /$ D.S.C. description (or any of the other geometrical models which are special cases of Bollmann's approach - such as the Planar Matching Model). $b$ ) From the stand point of boundary dependent properties, I believe it is now becoming clear that it is the non-equilibrium boundary defects which are of most consequence. These defects are describable as perturbation to the intrinsic structure.

Ph. MAITREPIERRE: The experiments that you described concerned precipitate nucleate at grain boundaries in materials thermomechanically treated. Would your conclusions about the respective role of extrinsic defects and G.B. structure be valid for simpler cases i. e. polycrystalline materials after soaking or homogenizing treatments ?

B. RALPH : In my oral presentation I mentioned our work on precipitate distributions (reference number 27 of the formal contribution). In my view the data I referred to illustrated the main thesis of my formal presentation - that is that the nonequilibrium boundary defect components are those which are of most importance in controlling boundary dependent properties in real materials. In my opinion this remark would be equally valid for the cases which you quote. In general, for instance extrinsic defects have much longer range strain fields than do the intrinsic components. Thus they 
have a more major role in attracting in impurities, etc... Soaking/homogenising treatments may contribute to an overall reduction in the concentration of non-equilibrium components of boundary structure.

D. MC LEAN : To improve stastitics of metallurgical structure I recommend using the $1 \mathrm{MV}$ electron microscope, which can examine up to $50 \mathrm{x}$ the volume of sample that can be examined by a $100 \mathrm{kV}$ microscope. At the NPL we have measured structural quantities with the same degree of reproduceability with which ordinary mechanical properties can be measured.

B. RALPH : I would agree that it is possible to study a larger volume of material with a $1 \mathrm{MV}$ electron microscope than with a conventioned $100 \mathrm{kV}$ machine although I regard your figure of $50 \mathrm{x}$ as somewhat high. For some cases I would agree that there would be a real advantage in using this expensive technique.

However, some disadvantages should also be noted. For instance image contrast is much more complicated in the million volt machine and it would far more difficult to perform our high resolution contrast analysis. Also, in these technological materials real problems could be encoun- tered due to the overlap of images from the various defect structures. In other words the extent of planar section of boundaries in our case is very small.

In my view, scanning transmission electron microscopy at 100-200 $\mathrm{kV}$ may prove much more useful in such studies than $1 \mathrm{MV}$ machine. Not only is the penetration good with these devices but it is possible to combine chemical analytical facilities and also to enhance contrast in weak beam mades electronically.

J. P. LANGERON : La précipitation en surface ou aux joints résulte, à mon avis, le plus souvent d'une chimisorption préalable à haute température. La précipitation se produit, lors du refroidissement. Elle est influencée par l'ensemble des dislocations présentes, au moment de la germination, dans le joint ; elle modifie à son tour les dislocations qui peuvent être observées après refroidissement.

B. RALPH : In general I agree with your comment. I would, however, stress that in my opinion it is the non equilibrium components (which have long range strain fields) which are largely responsible for attracting in solutes. The major of the intrinsic structure, as I see it, is in aiding the redistribution of solute within the boundary. 